\title{
Cognición, afectividad y resiliencia en el perdón de una transgresión en la pareja
}

\author{
Cognition, affectivity and resilience in the forgiveness of a transgression in the couple
}

\author{
Mónica Bolaños-Pasquel, ${ }^{1}$ María Judith López-Cárdenas, ${ }^{2}$ Brenda Guerrero, Jorge Cruz-Cárdenas, ${ }^{3}$ Carlos Ramos-Galarza-2
}

\section{Resumen}

La transgresión de los acuerdos entre la pareja es una problemática actual que genera malestar a nivel personal, familiar y social. En este artículo, se reporta una investigación que analiza un modelo que propone la interacción entre las variables cognición positiva, afecto positivo y resiliencia como factores predictivos que aportan en la comprensión del proceso de perdón de una transgresión en la pareja. En la investigación participaron 203 adultos (103 mujeres $60 \%$, 88 hombres $40 \%)$ entre 18 y 26 años de edad $(\mathrm{Medad}=20.4, \mathrm{SD}=1.68)$. El modelo hipotetizado fue testeado mediante el proceso de ecuaciones estructurales, encontrándose adecuados niveles de bondad de ajuste $\mathrm{x} 2=113.87, \mathrm{GL}=84, \mathrm{p}=.02, \mathrm{SRMR}=.05$, RMSEA $=.04(.03-.06)$, CFI $=.97$. Los resultados encontrados aportan en dos niveles esenciales en la línea de investigación de la interacción entre parejas, en primer lugar, a nivel teórico, se aporta con una teoría innovadora que permite comprender el proceso de perdón en la pareja y, en segundo lugar, a nivel clínico, para la intervención psicoterapéutica en variables como la cognición y afecto positivo que beneficiarían a que una pareja pueda superar un conflicto y llegar a un perdón.

Palabras clave: afecto positivo, cognición positiva, perdón, relación de pareja, resiliencia

\begin{abstract}
The transgression of the agreements between the couple is a current problem that generates discomfort on a personal, family and social level. In this article, an investigation is reported that analyzes a model that proposes the interaction between the variables positive cognition, positive affect and resilience as predictive factors that contribute to the understanding of the process of forgiveness of a transgression in the couple. 203 adults (103 women 60\%, 88 men 40\%) between 18 and 26 years of age participated in the research (Mage $=20.4, \mathrm{SD}=1.68$ ). The hypothesized model was tested through the process of structural equations, finding adequate levels of goodness of fit $\mathrm{x} 2=113.87, \mathrm{GL}=84, \mathrm{p}=.02$, SRMR $=.05$, RMSEA $=.04(.03-.06)$, CFI $=.97$. The results found contribute to two essential levels in the line of investigation of the interaction between couples, firstly, at a theoretical level, it is provided with an innovative theory that allows us to understand the process of forgiveness in the couple and, secondly, to clinical level, for psychotherapeutic intervention in variables such as cognition and positive affect that would benefit a couple to overcome a conflict and reach a forgiveness.
\end{abstract}

Keywords: positive affect, positive cognition, forgiveness, couple relationship, resilience

Rev. Ecuat. Neurol. Vol. 30, Nº 3, 2021

\section{Introducción}

Las relaciones de pareja, como cualquier otra interacción entre seres humanos, se ve afectada por diferentes factores, tal como la transgresión a los acuerdos implícitos y explícitos que rigen su día a día, siendo este el contexto en el cual se inserta la presente investigación. ${ }^{1,2}$

Diversos factores han generado que la interacción entre parejas en los últimos años aumenten actos de transgresión, por ejemplo, los diversos dispositivos tecnológicos y redes sociales que incrementan la comunicación, añadido a los cambios en el comportamiento humano, en donde diversos estándares culturales ven como poco atractivo presentar una conducta basada en el compromiso hacia el respeto hacia los diversos acuerdos de la pareja,
${ }^{1}$ Centro de Investigación en Mecatrónica y Sistemas Interactivos, Universidad Tecnológica Indoamérica.

${ }^{2}$ Facultad de Psicología, Pontificia Universidad Católica del Ecuador.

${ }^{3}$ Centro de Investigación ESTec, Universidad Tecnológica Indoamérica. Quito, Ecuador:
Correspondencia:

Carlos Ramos-Galarza

Facultad de Psicología, Pontificia Universidad Católica del Ecuador. Av. 12 de octubre y Roca.

Teléfono: +593998412108

ORCID: 0000-0001-5614-1994

E-mail: caramos@puce.edu.ec 
lo cual genera grandes dificultades en este tipo de interacciones. ${ }^{3}$ A nivel social, las relaciones de pareja pueden servir como centros de fortalecimiento de los roles sociales previamente aprendidos. Por ejemplo, la estabilidad de la pareja, en el contexto Latinoamericano, es un factor que fortalece los roles sociales y permite el desarrollo de la familia, que a su vez es el núcleo en el cual se forman los nuevos sujetos sociales. ${ }^{4}$

Al momento de hablar sobre una posible transgresión en una pareja, debemos remontarnos a la estructura social que determina la conformación de la misma y esta viene a ser la monogamia. Hay conocimientos equívocos sobre la definición de la monogamia, por ejemplo, se suele considerar que la misma es sinónimo de fidelidad, moralidad y que es universal. La monogamia comprende a la monoginia y a la monoandria, la primera significa que el hombre tiene una sola pareja por vez y la segunda que la mujer tiene una sola pareja a la vez. Ahora bien, ¿cuál fue la necesidad del mundo occidental en validar a la monogamia cómo un estilo de vida? Esta pregunta fue resuelta hace siglos por los griegos, considerados como los originarios de la civilización occidental, los mismos proclamaron los beneficios de tener una pareja a la vez para asegurar la fidelidad de la esposa y la consiguiente paternidad de los futuros herederos de sus bienes. De esta manera, se generan los primeros vestigios de la predominancia del hombre sobre la mujer, ya que, en un inicio, la monogamia tiene entre sus objetivos más importantes exigir la fidelidad de la mujer, ya que si la misma cometía adulterio era castigada severamente y considerada nada virtuosa y disoluta, mientras que, en el hombre el adulterio era considerado un desliz que ameritaba comprensión.

Las transgresiones ocurridas en épocas pasadas, en la mayoría de los países del mundo, no son castigados de la misma manera, ahora las personas pueden obtener una separación emocional y económica acorde a sus necesidades. Pero el agravio, ofensa o transgresión dentro de la pareja, desde hace siglos, viene siendo la problemática principal de la sobrevivencia de la monogamia, por lo que en la actualidad las nuevas generaciones consideran a la monogamia como un atentado a la libertad y buscan en otras formas de amor para protegerse de los conflictos. La transgresión ha sido definida como la violación percibida por un miembro de la relación, de las normas impuestas para la misma. Según McCullough, tras un error por cualquiera de las partes, se puede generar en la persona dos tipos de respuesta que le permitan alejarse de su transgresor, la primera es la evitación, la persona herida elude o evade a su pareja para no dar lugar a una conversación o respuesta a la transgresión ocurrida. El segundo tipo de respuesta es la venganza, a través de la cual, la víctima busca herir a su pareja ya sea física, psicológica o verbalmente. ${ }^{6}$
Cuando la pareja se encuentra en búsqueda de una reacción vengativa, los afectos y el perdón jugarán un papel fundamental en la continuidad de la pareja, ya que esto puede hacer que la misma se sienta menos motivada para tomar represalias contra el compañero que ofendió la relación, menos motivado a evitar o alejarse de la pareja y más motivación para buscar la consiliación con el o la transgresora. Por ello el trabajo psicoterapéutico en la relación de pareja estaría enfocado a los motivadores de union de la persona herida con su pareja. Por otro lado la gravedad de la transgresión, la reincidencia, la satisfacción y compromiso en la relación, también suelen ser motivos de la continuidad o no de la pareja.

La gravedad de la transgresión ocurrida dentro de la relación de pareja, podría convertirse en un factor decisivo en cuanto a la respuesta del individuo que forma parte del conflicto, en general, la evidencia empírica de los autores Waldron y Kelley? demuestra que es más probable dejar la relación cuanto mayor sea la gravedad percibida de la transgresión. Por otro lado, en relación con el género, la evidencia muestra que las mujeres perciben las transgresiones como más graves que los hombres, pero es la mujer quien encuentra mayor posibilidad de perdón hacía su pareja. ${ }^{8}$ De todos los tipos de transgresiones la infidelidad es considerada la más grave y la más dificil de perdonar ya que podría afectar la continuidad de la relación. Se ha visto que a pesar de la resiliencia, este fenómeno puede afectar a largo plazo el comportamiento de la pareja ya que viola las normas de exclusividad y fidelidad entre sus miembros. Cuando un individuo es reincidente en el mismo error o en otro agravio de las normas planteadas en la relación, dependerá de los factores que la persona herida encuentre como determinantes en su futuro de pareja, por ejemplo, si una persona se encuentra dentro de un círculo de violencia, el "perdón" se encontrará de manera más sencilla, por otro lado si la pareja tiene un buen autoconcepto y autoestima podrá estimar la necesidad de estár al lado de esa persona. Un factor determinante se ha visto en ocasiones en parejas que tienen hijos, el no separarse a pesar de la reincidencia por factores emocionales o económicos.

El modelo de Inversión propuesto por Rusbult, ${ }^{9}$ menciona que el compromiso de una persona hacia la relación se puede volver un predictor central en la desición de finiquitar o mantener la union a largo plazo, respondiendo a los conflictos de modos constructivos y olvidando las equivocaciones. ${ }^{10}$ Por otro lado, uno de los predictores que acompaña la variante del compromiso a la relación es la satisfacción en la misma, de este modo, si la unión viene siendo ya insatisfactoria y ocurre una transgresión, se minimiza el valor a largo plazo y se pone en juego la inversión ya sea emocional o económica que se ha vertido en la relación. Así, un incremento en la satisfacción de la vida de pareja podría llevar a un mayor compromiso con la 
misma, incrementando las posibilidades de reconciliación y disminuye las probabilidades de desersión. ${ }^{11}$

Las parejas enfrentan diferentes obstáculos durante los enfrentamientos, las variables mencionadas anteriormente pueden ser traducidas en la capacidad cognitiva y afectos positivos o negativos que tenga la parte herida para tomar desiciones en cuanto al accionar frente a la transgresión. Considerando la evidencia planteada hemos propuesto como investigadores la variable de la cognición y el afecto positivo como benefactores a que una pareja pueda superar un conflicto y llegar a un perdón.

Ahora bien, ¿Por qué la cognición y los afectos positivos vienen a ser tan importantes en la reconciliación o perdón después de una transgresión? La cognición nos permite como seres humanos el control del medio interno, la aprehensión del medio externo y la constitución de un Yo consciente, nos permite tratar la información que percibimos y ejecutar una acción, dentro de una relación afectiva. Al momento de una transgresión se pueden generar creencias o sesgos cognitivos que perjudiquen a la pareja, como por ejemplo: la inferencia arbitraria, se producen conclusiones demasiado rápidas sin evidencias claras al respecto; abstracción selectiva, la cual saca de contexto la información recibida resaltando los detalles negativos; sobregeneralización, una situación se vuelve un modelo permanente; magnificación o minimización, se atribuye mayor o menor importancia a un evento del que tiene realmente; etiquetación, se utilizan situaciones anteriores en donde se cometió un error para definir la incapacidad de la persona para resolver un nuevo error; pensamiento dicotónimo, personalización, entre otros sesgos cognitivos. ${ }^{12}$

Por otro lado, el afecto es una emoción compleja que necesita una adecuada regulación. El afecto positivo, refleja el punto hasta el cual una persona se siente entusiasmada, activa, alerta, enérgica y participativa, por otro lado, el afecto negativo representa una dimensión general de displacer, tristeza, poca energía, miedo, ira, culpa, nerviosismo, entre otros. Se puede decir entonces que para ayudar a una persona se sienta mejor y dispuesta a perdonar al transgresor, se necesitaría que los afectos positivos sean recalcados, de esta manera, los sesgos cognitivos no tendrían un amplio espacio para desarrollarse. Este algorítmo terapéutico en donde la cognición y los afectos positivos toman lugar, serán utilizados en parejas que deseen continuar juntos, utilizando el motivador de ir a consulta terapéutica como un plus en las variables a favor de la relación. A continuación, se pondrá en evidencia la necesidad de un plan terapéutico que tenga en cuenta la cognición y los afectos positivos en pro de la continuidad de la relación.

Según Raftar y Shareh ${ }^{12}$ debido a los problemas psicoemocionales que pasa una pareja herida por una transgresión, se recomienda utilizar terapia ligada al perdón para reducir los sufrimientos mentales y los problemas de comunicación. Según Apostolou y Demosthenous ${ }^{13}$ existen al menos cuatro razones por las que una persona perdona a su pareja; en ninguna de ellas se habla de la cognición o afecto positivo, generando un aporte científico con la propuesta presente. Wilkinson y Dunlop ${ }^{14}$ realizaron un estudio en donde demostraron que la forma de narración de la transgresión es un factor importante para el perdón; si analizamos la perspectiva de los autores, la forma de percepción que viene a ser una habilidad cognitiva, juega un rol importante en la capacidad de continuidad de la pareja. Para Coutinho y colaboradores ${ }^{15}$ fue importante explorar la presencia de un patrón de sincronía fisiológica en la actividad electrodérmica durante la interacción de pareja, esta actividad generaba afectos positivos en la misma; este hallazgo sumado con afectos positivos podría ser beneficioso para las parejas que han vivido transgresiones. Por otro lado, Schrage y colaboradores ${ }^{16}$ evidenciaron que la comunicación verbal y no verbal es crucial para la estabilidad de la pareja; estas formas de comunicación vendrán a ser esenciales en un proceso de perdón, adaptando a los afectos positivos como pilares en el camino terapéutico. Por otro lado, Retana-Franco y Sánchez-Aragón ${ }^{17}$ identificaron la relación entre los estilos de apego y las premisas histórico-socioculturales del rompimiento romántico, en donde juega en parte afectos negativos como el abandono, esta investigación resalta la necesidad de la presencia de afectos positivos ante la carga de la transgresión.

Gracias a las investigaciones mencionadas anteriormente, recalcamos como investigadores la necesidad del presente artículo. A continuación, se evidenciará el método y los instrumentos utilizados para justificar cómo la cognición positiva y afecto positivo benefician a las parejas que se encuentran en conflicto a llegar a un perdón.

\section{Método}

\section{Participantes}

Para la investigación se trabajó con una muestra de 203 adultos jóvenes de Quito, Ecuador. Su edad estuvo conformada entre 18 y 25 años $($ Media $=20.19, \mathrm{DE}=$ 1.17) La distribución según el género de los participantes fue de 113 mujeres (60\%) y 88 hombres $(40 \%)$. Todos los participantes contaban con un nivel educativo superior. El nivel socioeconómico fue medio y medio alto.

\section{Instrumentos}

Se utilizaron la escala de resiliencia de Wagnild y Youn adaptada a Latinoamérica ${ }^{18}$ Está conformada por 25 ítems que permiten valorar los componentes de la resiliencia: confianza en sí mismo, ecuanimidad, perseverancia, satisfacción personal y sentirse bien solo. Esta escala ha reportado adecuadas propiedades psicométricas en su aplicación en auto-reporte con niveles de consistencia interna entre $\alpha$ 
$=.79$ y $.81 .^{19} \mathrm{El}$ otro instrumento utilizado fue la escala de perdón y resentimiento hacia la pareja que permite valorar los componentes de benevolencia para el perdón, afecto y cognición positivos. Los valores de confiabilidad reportados en investigación previa sobre esta escala se encuentran en un rango entre $\alpha=.92$ y $.94 .^{18}$

\section{Procedimiento}

Se inició la investigación invitando a jóvenes universitarios a participar voluntariamente en el estudio. Posteriormente se entregó un formulario de consentimiento informado en donde se describía objetivos de la investigación y demás elementos éticos. Posterior a la firma del consentimiento de participación voluntaria, se entregaron los cuestionarios para ser completados por los participantes. Una vez obtenidos los instrumentos, se procedió a construir la base de datos, ejecutar los análisis estadísticos y escribir el presente reporte de la investigación. Este estudio tuvo la aprobación del Comité de Ética de la Universidad Tecnológica Indoamérica de Ecuador.

\section{Plan de análisis de datos}

Para esta investigación se utilizó el programa SPSS versión 25, en donde se realizaron los siguientes análisis estadísticos: descriptivos de tendencia central y dispersión, Alpha de Cronbach y correlación. En el paquete AMOS versión 23 se realizó el proceso de ecuaciones estructurales para analizar el modelo propuesto.

\section{Resultados}

En los descriptivos y consistencia interna de las variables, se encontró: perdón a la pareja $\mathrm{M}=11.18, \mathrm{SD}=2.37$, afecto positivo $\mathrm{M}=7.57, \mathrm{SD}=3.39$, cognición positiva $\mathrm{M}$ $=13.90, \mathrm{DE}=3.89$ y resiliencia $\mathrm{M}=21.15 \mathrm{SD}=3.59$.

Antes de realizar el análisis del modelo testeado, se analizó la confiabilidad y validez de cada variable valorada en su respectiva escala. Las variables perdón a la pareja $(\alpha=.80)$, cognición positiva $(\alpha=.73)$ y afecto positivo $(\alpha=.79)$ presentaron un adecuado valor de consistencia interna $(\alpha=.80)$. Para analizar su validez se procedió a realizar un análisis factorial confirmatorio de su configuración, encontrándose que los tres factores presentan un ajuste adecuado $\mathrm{x} 2=58.66, \mathrm{GL}=32, \mathrm{p}=.003, \mathrm{SRMR}=$ $.04, \mathrm{RMSEA}=.06(.03-.09), \mathrm{CFI}=.96$ (ver figura 1$)$.

En la variable de resiliencia se encontró un adecuado nivel de consistencia interna $(\alpha=.80)$ y su configuración factorial fue correcta $\mathrm{x} 2=4.88, \mathrm{GL}=5, \mathrm{p}=.44$, SRMR $=.02, \mathrm{RMSEA}=.01(.01-.09), \mathrm{CFI}=.96($ ver figura 2$)$.

Una vez que se contó con la adecuada validez y confiabilidad de las mediciones realizadas se procedió a testear el modelo hipotetizado en la investigación, en el cual se consideró como variable endógena el perdón a una transgresión, predicha por las variables exógenas

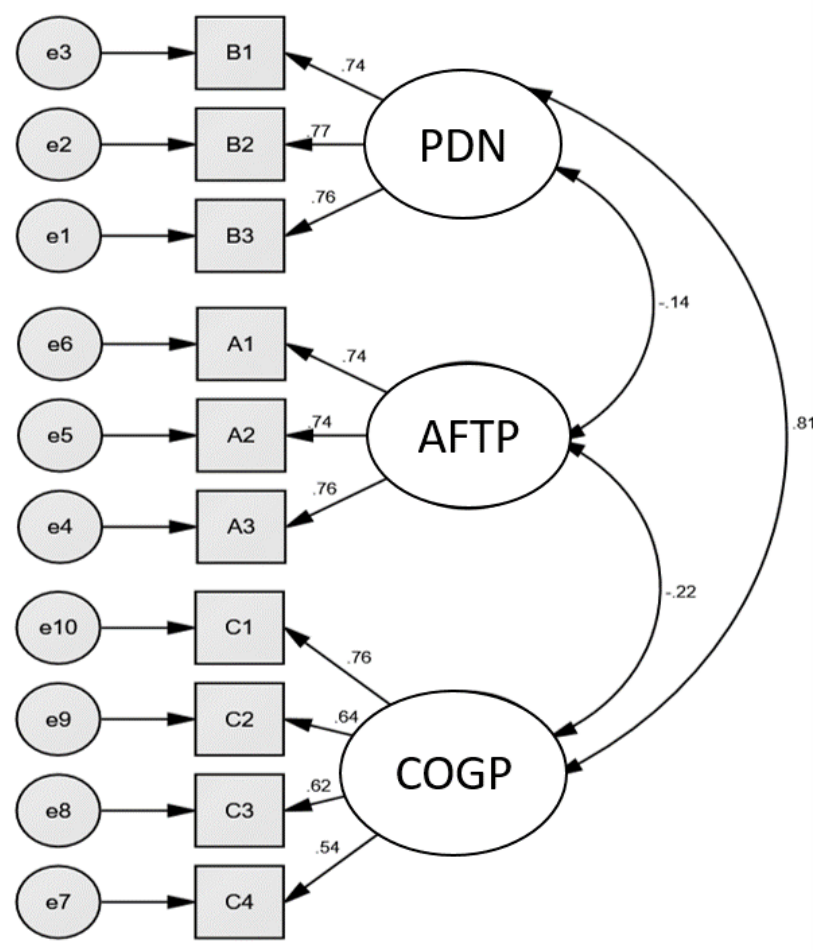

Figura 1. Confiabilidad y validez de las variables valorada en su respectiva escala.

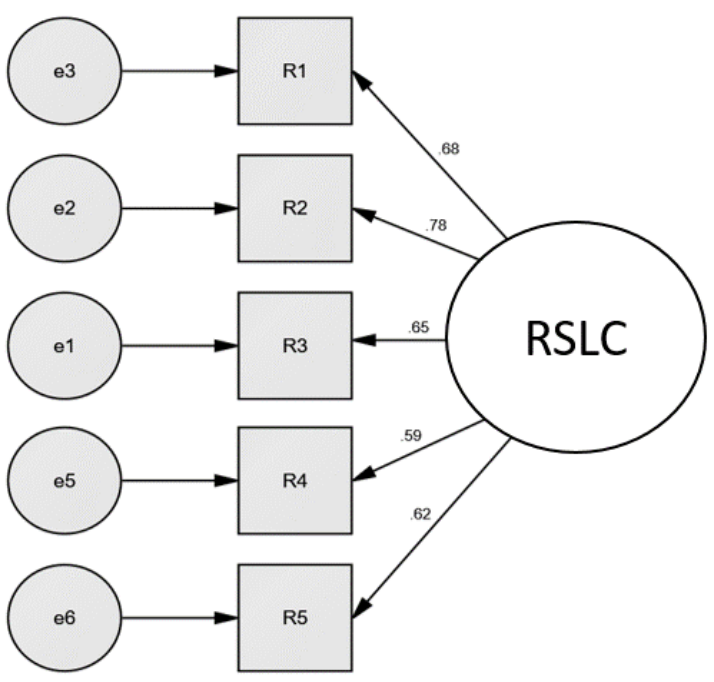

Figura 2. Consistencia interna de la variable de resiliencia. 
afecto positivo y cognición positiva mediadas por la resiliencia. Este modelo (figura 3 ) presentó como indicadores de bondad de ajuste $\times 2=113.87, \mathrm{GL}=84, \mathrm{p}=.02$, SRMR $=.05, \mathrm{RMSEA}=.04(.03-.06), \mathrm{CFI}=.97$.

Con la finalidad de analizar si el efecto directo de las variables exógenas cognición positiva, tendrían un efecto únicamente mediado por la resiliencia, se analizó un modelo en cual se eliminaba dicho efecto (figura 4), el cual no presentó indicadores adecuados de bondad de ajuste $\mathrm{x} 2=214.37, \mathrm{GL}=87, \mathrm{p}=<.001, \mathrm{SRMR}=.14$, RMSEA $=.09(.07-.10), \mathrm{CFI}=.86$, por lo que, se afirma la parsimonia y datos en favor del modelo hipotetizado.

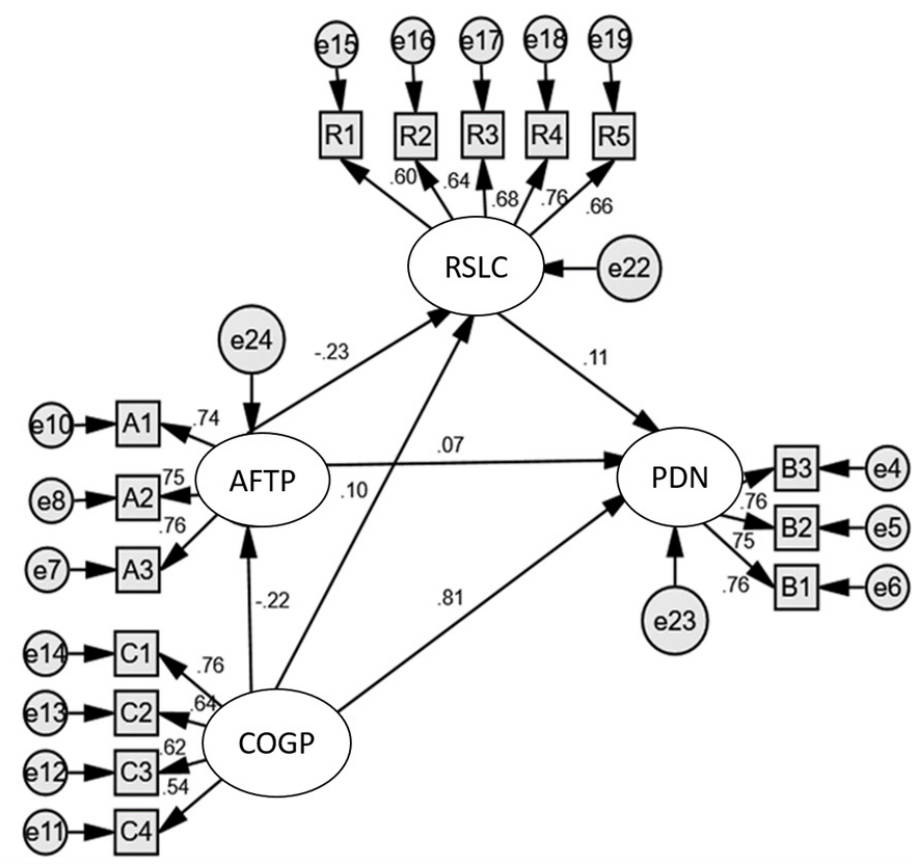

Figura 3. Validez y confiabilidad del modelo hipotetizado.

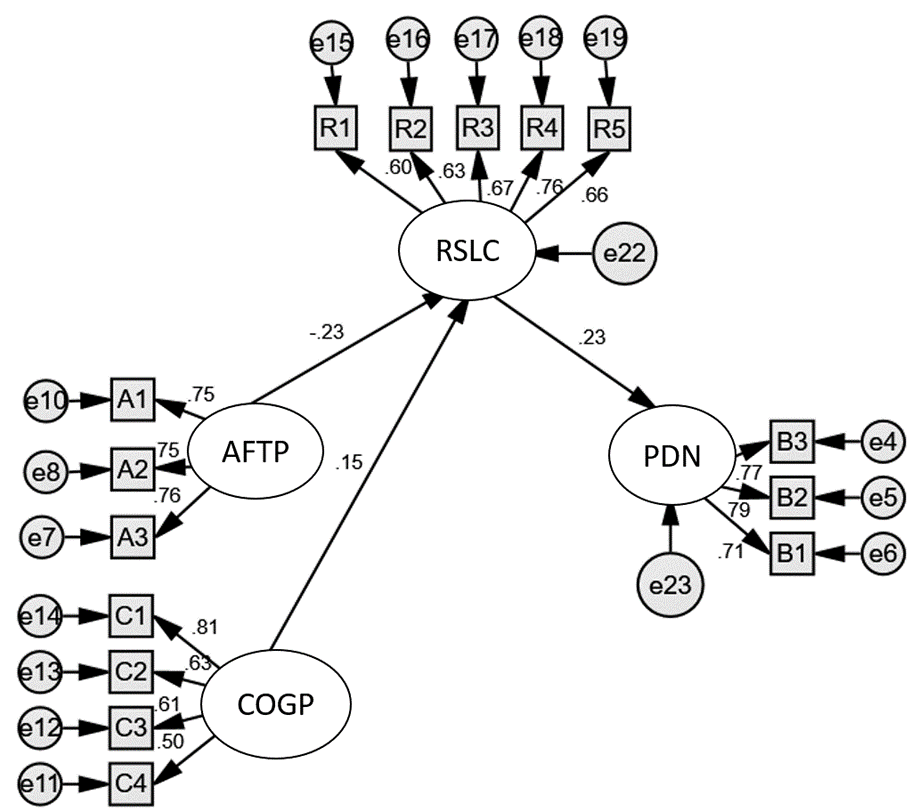

Figura 4. Análisis del modelo hipotetizado eliminando el efecto directo de las variables exógenas de cognición positiva. 


\section{Discusión}

El presente estudio propuso un modelo explicativo que tuvo como objetivo el brindar información agregada sobre los factores que rodean la reconciliación de la pareja en donde uno o ambos han cometido transgresiones. Para lograr este propósito se propuso un modelo teórico donde las variables de afecto positivo; cognición y resiliencia fueron considerados como factores causales del acto de perdonar. Los resultados obtenidos apoyan al modelo hipotetizado. El primer factor causal, la cognición positiva, puede concebirse como la actitud que adoptan los individuos ante las diferentes adversidades de la vida cotidiana; si esta variable está presente durante la confrontación y durabilidad del conflicto, la pareja podría tender a utilizar estrategias adaptativas para abordar la situación e intentar buscar soluciones en conjunto para evaluar positivamente la relación ayudando a su contexto. La cognición positiva influye en la capacidad de cada individuo para perdonar una transgresión haciendo que no caiga en sesgos cognitivos, entonces, viene a ser más probable que un individuo pueda observar un conflicto como un obstáculo que facilitaría la decisión de perdonar. Este factor tuvo un papel importante frente a las variables de afecto positivo y resiliencia ya que la cognición viene a jugar un papel de predictor del comportamiento de un individuo.

Por otro lado, el afecto positivo, como se ha mencionado anteriormente, puede entenderse como un rasgo personal estable que refleja las experiencias emocionales positivas de un individuo, el modelo hipotetizado, mostró que la variable afecto positivo tenía un efecto directo sobre el perdón y otro mediado por la variable resiliencia; de este modo si existiese un conflicto dentro de la pareja $\mathrm{y}$ el individuo ofendido tiene altos niveles de afecto positivo, esto puede influir en la decisión de perdonar la transgresión. Ahora bien, el factor resiliencia que ha jugado un papel fundamental en los afectos y la cognición, permitirá al individuo encontrar estrategias de adaptación cuando se enfrente a situaciones de alto estrés, tal cómo una transgresión amorosa. El modelo hipotetizado mostró que la variable resiliencia tuvo un efecto directo sobre el perdón, el cual fue influenciado por las otras dos variables, cognición y afecto positivo, teniendo como resultado que ambas variables afectan en el nivel de resiliencia el individuo, es decir, si la parte ofendida dentro de la pareja tiene altos niveles de cognición y afecto positivos, esto puede ayuda a aumentar los niveles de resiliencia y por consecuencia mejorar las posibilidades de elegir el camino hacia el perdón y restructuración de la pareja.

El modelo explicativo presentado en este estudio tiene varias implicaciones a nivel clínico, teórico y social. En el contexto clínico, contar con un modelo explicativo, que incluya las variables causales del proceso de perdón en las parejas, brindando un postulado para trabajar de forma individual dentro del proceso terapéutico.
En cuanto a la variable de cognición positiva, podría ser beneficioso abordar el razonamiento ligado a la búsqueda de soluciones alternativas y argumentar frente a lo que prevalece cuando existe daño en la relación, que es la cognición negativa o sesgos cognitivos. En cuanto a la variable de afecto positivo, se podría abordar a nivel personal, evaluando el autoestima y autoconcepto frente a las interacciones diarias con la pareja. Finalmente, dentro del factor de resiliencia, se denota la importancia de tener en cuanta el estado de las dos variables anteriores dado que ambas influyen en la resiliencia de la pareja. Los aspectos abordados dentro del presente artículo pueden ser de utilidad a la hora de decidir qué tipo de técnica terapéutica utilizar al momento de abordar a una pareja en conflicto por transgresión.

A nivel teórico, el modelo presentado permite una mejor comprensión del proceso de perdón dentro de una relación romántica, ya que, al conocer las variables involucradas en dicho proceso, se puede profundizar y comprender la importancia de estas si se busca en el contexto terapéutico, por parte de la pareja, la guía hacía el perdón. A nivel social, con ayuda de la conceptualización teórica y la evidencia empírica de la población seleccionada, nos permite conocer cómo miembros de una misma localidad consideran por su cultura y crianza, aspectos similares de lo que significaría una transgresión. Esto abre paso hacia otras líneas de estudio, en donde se permita comparar localidades y sus respuestas frente al significado de la transgresión.

Finalmente, se debe tener en cuenta las limitaciones del presente estudio, las cuales fueron, la determinación de la población y localidad. No fue obligatorio que los voluntarios a responder dicho cuestionario tengan pareja amorosa al momento y se debe poner a consideración el sesgo presente al utilizar cuestionarios de autoinforme, sin embargo, para contrarrestar este sesgo, se valuaron los parámetros de consistencia interna de las escalas aplicadas.

\section{Referencias}

1. A. Delgado. Género y conflicto en las relaciones de pareja heterosexuales: la desigualdad emocional. Cuestiones de Género: De La Igualdad y La Diferencia. 2013; 8: 165-181. https://doi.org/10.18002/ cg.v0i8.884.

2. B. Narváez, C. Parra, M. Alonso y A. Madera. Actitudes hacia el amor en relaciones románticas de jóvenes universitarios. Praxis \& Saber. 2017; 8(16): 155-178. https://doi.org/10.19053/22160159. v8.n16.2017.4596

3. T. Rodríguez-Salazar y Z. Rodríguez-Morales. El amor y las nuevas tecnologías: experiencias de comunicación y conflicto. Comunicación y Sociedad. 2016; 25: 15-41. 
4. J. Sangrador. Consideraciones psicosociales sobre el amor romántico. Psicothema. 1993; 5(1): 181-196.

5. M. Veiga. Matrimonio monogámico en la cultura occidental. Tesis Psicológica. 2016; 11(2): 158-167.

6. M. McCullough. Forgiveness. Who does it and how do they do it? Current Directions in Psychological Science. 2001; 10: 194-197. https://doi. org/10.1111\%2F1467-8721.00147

7. V. Waldron y D. Kelley. Forgiveness communication as a response to relational transgression. Journal of Social and Personal Relationships. 2005; 62(4): 416435.

8. K. Shumman y M. Ross. Why women apology more than men: Gender differences in thresholds for perceiving offensive behavior. Psychological Science. 2010 20: 1-7.

9. C. Rusbult. Commitment and Satisfation in Romantic Associations: A test of the investment model. Journal of Experimental Social Psychology. 1980; 16: 172-186.

10. L. Barón y G. Zapata. Los sesgos cognitivos: de la psicología cognitiva a la perspectiva cognitiva de la organización y su relación con los procesos de toma de decisiones gerenciales. Ciencia y Sociedad. 2017; 45(1): 31-47. https://doi.org/10.22206/cys.2018.v43i1. pp31-48.

11. M. Raftar y H. Shareh. Mindfulness-based schema therapy and forgiveness therapy among women affected by infidelity: A randomized clinical trial. Psychotherapy Research. 2021; 1-13. https://doi.org /10.1080/10503307.2021.1913294

12. M. Apostolou y A. Demosthenous. Why People Forgive Their Intimate Partners Infidelity: a Taxonomy of Reasons. Adaptive Human Behavior and Physiology. 2020; 1-18. https://link.springer.com/ article/10.1007/s40750-020-00153-1
13. D. Wilkinson y W. Dunlop. Both sides of the story: Narratives of romantic infidelity. Personal Relationships. 2020; 1-27. https://doi.org/10.1111/pere.12355

14. J. Coutinho, P. Oliveira-Silva, E. Fernandes, O. F. Gonçalves, D. Correia, K. Perrone Mc-Govern y W. Tschacher. Psychophysiological Synchrony During Verbal Interaction in Romantic Relationships. Family Process. 2018; 1-18. https://doi.org/10.1111/ famp. 12371

15. K. Schrage, J. Maxwell, E. Impett, D. Keltner y G. MacDonald. Effects of Verbal and Nonverbal Communication of Affection on Avoidantly Attached Partners Emotions and Message Receptiveness. Personality and Social Psychology Bulletin. 2020; 1-14 https://doi.org/10.1177/0146167220910311

16. B. Retana-Franco y R. Sánchez-Aragón. El peso del apego y de la cultura en las estrategias de rompimiento amoroso percibidas por los abandonados. Acta Colombiana de Psicología. 2020; 23(1): 53-65. https://www.doi.org/10.14718/ACP.2020.23.1.4

17. A. Novella, Incremento de la resiliencia luego de la aplicación de un programa de psicoterapia breve en madres adolescentes, Lima: Universidad Nacional Mayor de San Marcos, 2002.

18. R. Rosales-Sarabia, S. Rivera, L. Reidl y M. García. Perdón y Resentimiento hacia la Pareja: Desarrollo y Validación de Dos Escalas. Revista Iberoamericana de Diagnóstico y Evaluación Psicológica. 2018; 47(2): 141-156 http://dx.doi.org/10.21865/ RIDEP47.2.10

19. E. Finkel, C. Rusbult, M. Kumashiro y P. Hannon. Dealing with betrayalin close relationships: Does commitment promote forgiveness? Journal of Personality and Social Psychology. 2002; 28: 956-974. 